\title{
MIGRATION
}

\section{Die Chancen nutzen}

VON RÜDIGER FRITZ UND

RAINER OHLIGER

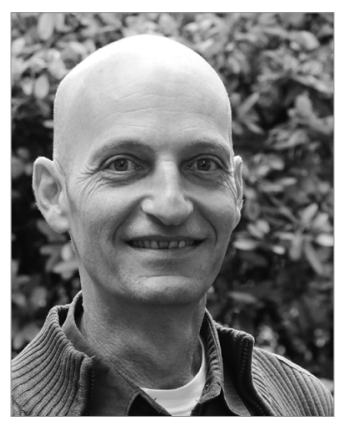

Rüdiger Fritz arbeitet im

Generalsekretariat des

Deutschen Roten Kreuzes als Referent zum Thema Integration von Geflüchteten. Er befasst sich im Schwerpunkt mit der Verbesserung von Strukturen für die Integration in Arbeit und der Förderung der Zusammenarbeit unterschiedlicher Akteure. www.drk.de

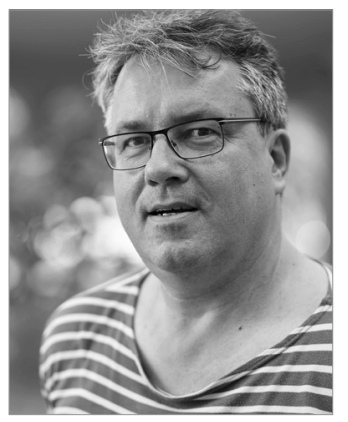

Rainer Ohliger leitet im Auftrag der Robert Bosch Stiftung das Programmbüro des Förderprogramms "Land.Zuhause. Zukunft - Integration und Teilhabe von Neuzuwanderern in ländlichen Räumen«.

Das Programm befindet sich derzeit in einer Pilotphase, an der sechs Landkreise beteiligt sind. www.land-zuhause-zukunft.de

\section{Die Integration von Neuzuwanderern könnte insbesondere für ländliche Gebiete eine Chance bieten.}

Die Debatte über die Aufnahme von Asylsuchenden in Deutschland ist zuletzt ein wenig ruhiger geworden. Die Frage, wie die Integration der Menschen, die in Deutschland bleiben werden, besser gestaltet werden kann, ist dagegen weiterhin hoch aktuell. Dabei geht es auch darum, wo Integration besser gelingen kann: In den vielfältigen Metropolen oder in Kreisen mit günstigen Mieten und Wohnungsleerstand.

Nicht wenige ländliche Regionen haben ein Interesse, Geflüchtete zu integrieren, um der negativen Bevölkerungsentwicklung entgegenzuwirken. Dagegen stehen viele Metropolen aufgrund der ohnehin angespannten Wohnungsmärkte deutlich unter Druck. Es könnte also eine Win-win-Situation sein, wenn sich mehr Menschen und Familien mit Flucht- oder Migrationsgeschichte für ein Leben auf dem Land entscheiden.

Die Praxis zeigt aber, dass es häufig Gründe gibt, die dazu führen, dass sich auch die neuen Nachbarn nach nicht allzu langer Zeit auf den Weg in die Städte machen. Um dem entgegen zu wirken und das Land attraktiver zu gestalten (auch für die ansässige Bevölkerung), braucht es Ideen, Mut und ein Bekenntnis zu kultureller Vielfalt.

\section{Demografische Entwicklung und Strukturwandel}

Wenn eine nachhaltige Integration gelingt, kann Zuwanderung (auch) als Chance für Regionen gesehen werden, aus denen immer mehr junge Menschen wegziehen. Bundesweit unterstützen vor allem die großen Wohlfahrtsverbände die Integration - rein ehrenamtliche Initiativen, wie sie in den Jahren 2014 bis 2016 entstanden sind, haben sich zu einem großen Teil wieder zurückgezogen.
Die Angebote der Wohlfahrtsverbände umfassen unter anderem Asylverfahrensberatung, Kinderbetreuung, Übergangsbegleitung zu Schulen, psychotherapeutische Angebote sowie die Jugendmigrationsdienste und Migrationsberatung für Erwachsene.

Viele der in der Flüchtlingsarbeit der letzten Jahre entstandenen Strukturen und Initiativen stehen aber vor einer ungewissen Zukunft oder sind bereits wieder verschwunden. Die Überführung in Regelstrukturen konnte häufig nicht realisiert werden. So fehlen künftig Angebote, wie zum Beispiel eine lokale Ehrenamtskoordination oder Integrationslotsen, die für die Einbindung von Zuwanderern benötigt werden.

\section{Verteilung von geflüchteten Menschen}

Die Bundesregierung hatte 2016 im Aufenthaltsgesetz eine Wohnsitzregelung beschlossen, die zunächst eine Verpflichtung zur Wohnsitznahme in dem Bundesland führt, in dem das Asylverfahren durchgeführt wurde - jedenfalls so lange die Betroffenen von Sozialleistungen abhängig sind, maximal aber für drei Jahre.

Gleichzeitig wurde den Bundesländern die Möglichkeit eröffnet, die Auflage auf einen bestimmten Ort - oder einen bestimmten Ort ausschließend - zu erweitern (Wohnsitzauflage), wenn damit das Ziel »einer nachhaltigen Integration in die Lebensverhältnisse der Bundesrepublik Deutschland " verbunden ist $(\mathbb{S}$ 12a, Absatz 3 AufenthG).

Die Frage ist, ob die Zuweisung zu einem bestimmten Wohnort auch den integrativen Bedürfnissen der Menschen gerecht wird und eine entsprechende Infrastruktur an diesem Ort vorhanden ist. Eine Studie des Sachverständigenrats deutscher 
Stiftungen für Integration und Migration und der Robert Bosch Stiftung aus 2017 schlägt vor, die Teilhabeperspektiven bei der Verteilung stärker zu berücksichtigen:

»Die Studie macht deutlich: Je besser die individuellen Voraussetzungen, Bedarfe und Zukunftspläne der Asylsuchenden und der zugewiesene Wohnort zusammenpassen, umso besser können die Menschen dort Fuß fassen. So kann auch verhindert werden, dass sie als anerkannte Flüchtlinge später weiterwandern und sich in bestimmten Regionen konzentrieren. [...] Statt die Asylsuchenden nur nach demografischen Strukturdaten zu verteilen, sollten integrationsrelevante individuelle und regionale Voraussetzungen aufeinander abgestimmt werden.« (1)

Die Heinrich Böll Stiftung hat im Februar 2019 eine Erweiterung dieser Idee für ein europäisches Verteilverfahren veröffentlicht. (2) Bei diesem so genannten »kommunalen Relocation-Mechanismus « würde ein EU-weites MatchingVerfahren bei Schutzsuchenden mit hoher Bleibeperspektive die Präferenzen von Kommen und Schutzsuchenden zusammenführen. Die Kommunen würden dabei mit ihrem spezifischen Profil und der Einbeziehung lokaler Unterstützergruppen ein Integrationsangebot erstellen, zu dem die Interessen der Geflüchteten zugeordnet würden um eine gezielte Verteilung zu ermöglichen.

\section{Kommunale Entwicklung initiieren und begleiten}

Die Robert Bosch Stiftung hat für die Jahre 2018/19 das neue Förderprogramm »Land.Zuhause.Zukunft - Integration und Teilhabe von Neuzuwanderern in ländlichen Räumen« aufgelegt. An dem Programm sind in der Pilotphase sechs Landkreise beteiligt: Coburg, Goslar, Harz, Ludwigslust-Parchim, Prignitz, Vogtlandkreis.

Es werden themenspezifische Beratungs- und Begleitungsprozesse, Modellprojekte und Förderungen von Mikroaktivitäten zur Integration und Teilhabe von Neuzuwanderern durchgeführt. Ziel ist es, bedarfsorientierte Lösungen zu entwickeln und zu erproben, die Antworten auf die (relativ) neue Herausforderung der Migration im ländlichen Raum liefern können.

Im Jahr 2016 wurde dafür eine Vorstudie und Bedarfsanalyse (3) erstellt, auf deren Grundlage, die verschiedenen

Module des Programms im Dialog mit kommunalen Akteuren konzipiert und umgesetzt wurden. Als zentrale Themenfelder des Programms und der Herausforderungen im ländlichen Raum kristallisierten sich folgende Fragen heraus:

Wie gelingt die langfristige Bindung
im ländlichen Raum?

7 Welche institutionellen und kon2. zeptionellen Herausforderungen gibt es für die Integration und Teilhabe von Migranten auf kommunaler Ebene?

3 (Wie) Kann Migration einen Bei3. trag zur Ausgestaltung des demographischen Wandels (Schrumpfung, Alterung) auf dem Lande leisten?

Welche praktischen Probleme 4. sind zentral für die Bindung, Integration und Teilhabe von Migranten im ländlichen Raum (z. B. Mobilität, Öffnung der Zivilgesellschaft, Zugang zum Arbeitsmarkt, vor allem ins duale System)?

Folgende konkrete Vorhaben wurden in den sechs beteiligten Landkreisen initiiert:

- Gezielte Steuerung von Integrationsarbeit durch abgestimmte integrationspolitische kommunale Konzepte und Politiken: Entwicklung kommunaler, Integrations- und Teilhabekonzepte, auch in dezentralisierter Form; Entwicklung eines Machbarkeitskonzeptes für ein kommunales, individuelles Integrationsmonitoring (4)

- Beidseitige Begleitung von Betrieben und Geflüchteten beim Zugang zum ländlichen Arbeitsmarkt

- Bindung von Geflüchteten und Gewinnung von Neuzuwanderern für Pflegeberufe im ländlichen Raum

- Ausbau und Vertiefung von Integrationsangeboten im Bereich Sprachvermittlung (Zusatzmodule)

- Etablierung von Zugangswegen in die Zivilgesellschaft (kommunales Vereinswesen).

Der Prozess der Konzeptentwicklung und Konzeptumsetzung lief in den beteiligten Landkreisen bis Juni 2019. (5) Die Ergebnisse der Pilotphase zeigen, dass es im ländlichen Raum vielerorts einen ungedeckten Beratungsbedarf bei den Fragen Migration, Integration und Teilhabe gibt.

\section{Fazit}

Aufgrund der beschriebenen Entwicklungen in vielen ländlichen Regionen sollte die Zuwanderung von Menschen mit Flucht- und Migrationsgeschichte als Chance verstanden werden. Dazu müssen der Zugang zu Bildung und Arbeit, die Erreichbarkeit von Kontakt- und Begegnungsmöglichkeiten sowie ein Konzept für Mobilität über den Sozialraum hinaus geschaffen werden. Ebenso müssen zivilgesellschaftliche Initiativen und Angebote in die Lage versetzt werden, dauerhaft angelegte Strukturen für das Miteinander vor Ort zu schaffen.

Die Verteilung von Asylsuchenden und anerkannt Schutzberechtigten ergibt derzeit allerdings keine zuverlässige Planungsgrundlage für die Kommunen. Die angesprochenen Modelle einer bedarfsorientierten Verteilung, bei der sowohl die Integrationsvoraussetzungen der Schutzsuchenden als auch die der aufnehmenden Kommunen berücksichtigt würden, sollten weiter diskutiert und erprobt werden.

\section{Anmerkungen}

(1) Forschungsbereich beim Sachverständigenrat deutscher Stiftungen für Integration und Migration: Wie gelingt Integration? Asylsuchende über ihre Lebenslagen und Teilhabeperspektiven in Deutschland. Eine Studie des SVR-Forschungsbereichs und der Robert Bosch Stiftung, Berlin, 2017.

(2) Der Weg über die Kommunen. Empfehlungen für eine neue Schlüsselrolle der Kommunen in der Flüchtlingsund Asylpolitik der EU. 2. überarbeitete Auflage, Februar 2019.

(3) www.bosch-stiftung.de/de/publikation/auf-dem-weg-zur-fluechtlingsintegration-laendlichen-raeumenergebnisse-einer.

(4) Für die Kurzexpertise zum Thema kommunales individuelles Integrationsmonitoring siehe: www. bosch-stiftung.de/sites/default/files/ publications/pdf/2019-02/KurzExpertise_Kommunales_individuelles_Integrationsmonitoring_KiIM. pdf.

(5) Über eine Fortführung des Programms kann man sich auf der Seite der Robert Bosch Stiftung informieren: www.bosch-stiftung.de. 